\title{
Unpolished Thai Rice Prevents ACF Formation and Dysplastic Progression in AOM-Induced Rats and Induces Apoptosis Through Redox Alteration in CaCo-2 Cells
}

\author{
Achiraya Tammasakchai ${ }^{1}$, Chaiyavat Chaiyasut ${ }^{2}$, Suda Riengrojpitak ${ }^{1}$, Prasit \\ Suwannalert ${ }^{1 *}$
}

\begin{abstract}
Oxidative stress is associated with colon carcinogenesis including aberrant crypt foci (ACF) formation and it plays an important role in pathophysiological changes in cancer cells. The aims of this study were to investigate the effects of dietary unpolished Thai rice (UTR) on ACF formation and dysplastic progression in azoxymethane (AOM)-treated rats. Anti-cancer efficacy of UTR regarding apoptotic induction and oxidative redox status in human colon cancer $(\mathrm{CaCo}-2)$ cells was also investigated. Rats given $20 \%$ and $70 \%$ of UTR in the diet showed significantly and dose-dependently decreased total number of ACF. UTR treatment also was strongly associated with the low percentage of dysplastic progression and mucin depletion. In addition, we found that UTR significantly induced cancer cell apoptosis, increased cellular oxidants, and decreased the level of GSH/GSSG ratio in CaCo-2 cells. Our study suggests that UTR supplementation may be a useful strategy for CRC prevention with the inhibition of precancerous progression, with induction of cancer cell apoptosis through redox alteration.
\end{abstract}

Keywords: Aberrant crypt foci - oxidative stress - glutathione - apoptosis - unpolished Thai rice

Asian Pac J Cancer Prev, 16 (7), 2827-2832

\section{Introduction}

Aberrant crypt foci (ACF) are a precancerous lesion of colorectal cancer (CRC) which appear as the larger crypts and thicker layer of epithelial linings with slightly elevate and protrude toward the lumen (Bird and Good, 2000). Oxidative stress is an imbalance between the production of free radicals and an eliminating system (Lobo et al., 2010), it is widely accepted as a major cause of CRC development (Perse, 2013). In addition, many types of cancer cells required a certain level of oxidative stress to maintain the balance of cell proliferation and apoptosis (Noda, 2001; Loo, 2003). Interestingly, the excessive level of oxidative stress above a certain threshold can cause apoptotic induction in various cancer cells (Pelicano et al., 2004). Glutathione is an important antioxidant, it presents in reduced (GSH) and oxidized (GSSG) forms (Dickinson and Forman, 2002). GSH/GSSG ratio acts as a major thiol redox system (Aw, 2003; Circu and Aw, 2012) which plays a crucial role in cellular function (Traverso et al., 2013). The low level of GSH/GSSG ratio during oxidative stress may associate with cellular oxidants related to cancer cell death (Halliwell, 1994; Traverso et al., 2013).

Phytophenolics are widely distributed in various plants, they contribute to prevent or reduce oxidative stress (Shahidi et al., 1992; Basu et al., 2012). Previously, red color strain of unpolished Thai rice (UTR) showed a high antioxidant with phenolic compounds (Rattanachitthawat et al., 2010). The rats consuming UTR had a low level of malondialdehyde (MDA) - oxidative stress marker (Suwannalert et al., 2010). In addition, UTR treated rats also reduced total density and crypt multiplicity of ACF in AOM-induced rats (Tammasakchai et al.,2012). Although UTR has been recognized to prevent ACF formation, the effects of UTR on precancerous development and cellular oxidants were not determined. The aims of this study were to determine the effects of UTR on ACF formation and their histopathological changes in AOM-induced rats. The effects of UTR on cellular apoptosis, cellular oxidants, and redox alteration through GSH/GSSG ratio in human colon cancer $(\mathrm{CaCo}-2)$ cells were also investigated.

\section{Materials and Methods}

\section{Samples and reagents}

Dulbecco's modified eagle medium (DMEM), nonessential amino acid, L-glutamine, 3-(4,5-dimethylthiazol2-yl)-2,5-diphenyltetrazolium bromide (MTT), 2'-7'-dichlorodihydrofluorescein diacetate (DCFH-DA), bisBenzimide H 33342 trihydrochloride (Hoechst 33342), L-glutathione reduced, and L-glutathione oxidized were purchased from Sigma-Aldrich (St. Louis, MO). Fetal 
Achiraya Tammasakchai et al

bovine serum (FBS) and penicillin - streptomycin were obtained from Gibco (Invitrogen, USA).

\section{Animal design}

Male Sprague-Dawley rats were purchased from National Laboratory Animal Centre, Mahidol University, Nakhon Pathom, Thailand. The rats were randomly divided into 4 groups (6 rats/group); untreated group, AOM-induced rats without UTR treatment (AOM group), AOM-induced rats with UTR treatments of low dose $(\mathrm{AOM}+\mathrm{LD})$ and high dose (AOM+HD). Rats in untreated- and AOM-groups were fed with standard diet, while the AOM+LD and AOM+HD groups were fed with standard diet which containing $20 \%$ and $70 \%$ of UTR, respectively. The rats in groups of AOM, AOM+LD and $\mathrm{AOM}+\mathrm{HD}$ were subjected to subcutaneous injection of $\mathrm{AOM}$ at a dose of $15 \mathrm{mg} / \mathrm{kg}$ body weight at week 4 and 5 after starting experiment. At week 38 , all animals were sacrificed and their colons were collected. Colons were fixed in $10 \%$ buffered formalin for at least $24 \mathrm{~h}$ before ACF determination. Ethical approval for this study was obtained from CMU-ACUC:R3/2554.

\section{Aberrant Crypt Foci (ACF) determination}

The formalin-fixed colonic tissues were stained in $0.1 \%$ methylene blue solution for $5 \mathrm{~min}$ and observed the total numbers of ACF under a light microscope. The morphological characteristics of ACF were described as the crypts with darker staining, enlarged crypt, and elevated epithelial lining with slit-like lumens (Bird, 1995).

\section{ACF grading}

For histological evaluation, the paraffin blocks of colonic tissues were processed and sectioned serially at 4 $\mu \mathrm{M}$ thickness. The sections were stained with hematoxylin and eosin ( $\mathrm{H}$ and $\mathrm{E})$. According to the previous study (Xiao et al., 2008), ACF were classified into three categories including $i$ ) hyperplastic ACF (elongated, no dysplasia), ii) low-grade dysplastic ACF (elongated, slightly crowded and pseudostratified nuclei, well polarity, and normal or slightly decreased number of goblet cells), and iii) high-grade dysplastic ACF with elongated, crowded and pseudostratified nuclei, markedly increased N/C ratio, and significantly decreased number of goblet cells.

\section{ACF with mucin depletion determination}

The sections adjacent to $\mathrm{H}$ and $\mathrm{E}$ sections were then stained with Periodic acid-Schiff (PAS) stain for mucin determination. Mucin-producing cells with PAS staining were obtained in ACF. According to the method modification of previous study (Uchida et al., 1997), mucin depletion in ACF were classified into three categories including $i$ ) ACF with non-mucin depletion (normal production of mucin, no differences in PASpositive staining in entire ACF compared to adjacent normal crypts), ii) ACF with mild mucin depletion (mucin producing cells with PAS-positive staining presence more than half of the crypt), and iii) ACF with severe mucin depletion (mucin producing cells with PAS-positive staining presence less than half of crypt).
Unpolished Thai rice (UTR) extract for cell culture

Red color strain of unpolished Thai rice (Oryza sativa L.) was grounded by an electric blender and extracted with $95 \%$ ethanol by 1:5 (w/v). The extract was centrifuged at $3000 \mathrm{rpm}$ for $15 \mathrm{~min}$ and filtrated with polytetrafluoroethylene (PTFE) filter nylon $0.45 \mu \mathrm{m}$. The solvent was removed by rotary evaporator (R-200, Buchi, Switzerland). Then the sample extract was lyophilized (Super-Modulyo, Freeze Dryer) and re-dissolved with DMEM medium containing 0.1\% DMSO. The solution was kept in $-20^{\circ} \mathrm{C}$ until use.

\section{CaCo-2 Cell culture}

Human colon cancer (CaCo-2) cells lines were kindly provided by Prof. Dr. Nateetip Krishnamra, Center of Calcium and Bone Research (COCAB), Faculty of Science, Mahidol University, Thailand. Cells were routinely cultured in completed DMEM medium containing $15 \%$ fetal bovine serum, $1 \%$ non-essential amino acid, $1 \%$ L-glutamine, and $1 \%$ penicillin-streptomycin. Cells were maintained in $37^{\circ} \mathrm{C}$ and supplemented with $5 \% \mathrm{CO}_{2}$ in humidified incubator.

\section{Cytotoxicity test}

The MTT assay was used to evaluate the cytotoxicity of UTR extract. CaCo- 2 cells were seeded into 96-well plates at a density of $2 \times 10^{4}$ cells per well and maintained in $37^{\circ} \mathrm{C}$ and $5 \% \mathrm{CO}_{2}$ incubator for $48 \mathrm{~h}$. Cells were treated with UTR extract at various concentrations $(1,2.5,5,7.5$, 10 , and $12.5 \mathrm{mg} / \mathrm{ml}$ ) for $24 \mathrm{~h}$. The conditioned medium was removed and cells were washed with PBS buffer, $\mathrm{pH}$ 7.4. Then $200 \mu 1$ of serum free medium containing $0.5 \mathrm{mg} / \mathrm{ml}$ of MTT solution was added into each well and incubated at $37^{\circ} \mathrm{C}$ for $3 \mathrm{~h}$. The medium was removed and $200 \mu \mathrm{l}$ of DMSO was added to completely dissolve the precipitation. The absorbance was detected at $570 \mathrm{~nm}$ with a microplate reader (1420 Victor 2, Wallac, USA), results were expressed as the percentage of viable cells.

\section{Apoptotic induction test}

The apoptotic cells with nuclear condensation and fragmentation can be obtained by special staining with Hoechst 33342 that obtained under the fluorescence microscope (Allen et al., 2001). Caco- 2 cells were seed at $1 \times 10^{6}$ cells per well and grown on glass cover slips in 6-well plates and maintained for $48 \mathrm{~h}$. Cells were treated with UTR extract at various concentrations ( 5 and $7.5 \mathrm{mg}$ / $\mathrm{ml}$ ) for $24 \mathrm{~h}$. Cells were washed twice with PBS, fixed with methanol at $-20^{\circ} \mathrm{C}$ for $10 \mathrm{~min}$ and incubated with 1 $\mu \mathrm{g} / \mathrm{ml}$ Hoechst 33342 at $25^{\circ} \mathrm{C}$ for $20 \mathrm{~min} .200$ cells in four random fields were counted and data were expressed as percentage of apoptotic cells.

\section{Cellular oxidants}

Cellular oxidants were measured by fluorescence probes DCFH-DA as described in the modified method (Khan et al., 2013). $2 \times 10^{4}$ cells of CaCo-2 cells were seeded into the 96-well plates and maintained for $48 \mathrm{~h}$. Cells were incubated in completed DMEM medium with 5 and $7.5 \mathrm{mg} / \mathrm{ml}$ of the UTR extract for $24 \mathrm{~h}$. The treated cells were washed with PBS and incubated with serum free 
Unpolished Thai Rice Prevents ACF Formation and Dysplastic Progression in AOM-Induced Rats and Induces Apoptosis

medium containing $20 \mu \mathrm{M}$ of DCFH-DA for $1 \mathrm{~h}$ at room temperature in dark condition. The DCF fluorescence intensity was immediately assessed for cellular oxidants at excitation/emission wavelengths of $485 / 535 \mathrm{~nm}$ by using the fluorescence microplate reader (1420 Victor 2, Wallac, USA).

\section{Cellular glutathione determination}

The intracellular GSH and GSSG were determined by using a reverse-phase of high performance liquid chromatography (HPLC) (Waters 2695, Alliance, USA). CaCo- 2 cells $\left(5 \times 10^{7}\right.$ cells) were cultured in $75-\mathrm{cm} 2$ tissue culture flasks and maintained for $48 \mathrm{~h}$. Cells were treated with UTR extract at 5 and $7.5 \mathrm{mg} / \mathrm{ml}$ for $24 \mathrm{~h}$. Cells were washed with ice-cold PBS and detached by trypsinization, and lysed quickly by freeze-thaw method. The lysate was immediately deproteinated by adding the ice-cold of $5 \%$ metaphosphoric acid (MPA). The mixture was then incubated at room temperature in dark condition for 15 min and then centrifuged $(12,000 \mathrm{~g})$ at $4^{\circ} \mathrm{C}$ for $10 \mathrm{~min}$. The supernatant was kept and determined the GSH and GSSG forms by using the modified method (Hahn et al., 2007), $10 \mu \mathrm{l}$ of sample was injected into a column (4.6 mm X 250 $\mathrm{mm}$, ODS-80 TM, TOSHO, Japan). $0.1 \%$ trifluoroacetic acid (TFA) and $40 \%$ acetonitrile (ACN) were used as the mobile phase. The GSH and GSSG levels were identified by the absorbance spectra in the UV length at $210 \mathrm{~nm}$ with PDA detector. The commercial standards of L-glutathione reduced form $(\mathrm{GSH})$ and L-glutathione oxidized form (GSSG) were used as the references.

\section{Statistical analyses}

All results were presented as Mean \pm standard deviation (SD). The differences among groups were obtained by one-way analysis of variance (ANOVA). Effects of the treatments in different categories were analyzed by chisquare test. Statistical significance was considered at $\mathrm{p}<0.05$ with SPSS version 16 computer software.

\section{Results}

Dietary UTR inhibited ACF formation in AOM-induced rats

Total number of ACF in untreated group, AOMinduced rats, and UTR treated rats are shown in Figure 1. No ACF formation was found in untreated group. The AOM-induced rats (AOM group) had significantly increased the total number of $\operatorname{ACF}(56.25 \pm 8.77, \mathrm{p}<0.001)$ when compared with untreated group. Interestingly, the treated AOM-induced rats with 20\% UTR (AOM+LD group) and $70 \%$ UTR (AOM+HD group) were dose dependently decreased the total number of ACF at $23.67 \pm 8.09(\mathrm{p}<0.05)$ and $9.67 \pm 4.72(\mathrm{p}<0.01)$, respectively.

\section{Dietary UTR inhibited the formation of dysplastic ACF}

In this study, ACF were found in AOM, AOM+LD, and AOM+HD groups. All ACF formation can be classified into three grades with hyperplasia (Figure 2A), low-grade dysplasia (Figure 2B) and high-grade dysplasia (Figure 2C). The percentage of each ACF category is summarized in Table 1. The AOM group had high total percentage with $81.81 \%$ of dysplastic ACF. Dietary UTR treatment at $20 \%$ (AOM+LD group) and 70\% (AOM+HD group) showed dose dependently decreased the dysplastic ACF at $78.13 \%$ and $57.14 \%$, respectively. Generally, a high-grade dysplasia is well recognized with cancer development. In this study, the UTR treated groups showed strongly decreased the high-grade dysplasia at $34.38 \%(\mathrm{p}<0.05)$ and $21.43 \%(\mathrm{p}<0.001)$ in AOM+LD and AOM+HD groups, respectively when compared with untreated rats (AOM group). Therefore, the dietary of UTR may play a crucial role in the inhibition of dysplastic development.

Dietary UTR prevented mucin depletion in dysplastic ACF

Normally, the loss of mucin is associated with a degree of dysplastic ACF. In this study, all dysplastic ACF were classified into 3 grades with non-mucin depletion (Figure 2D), mild-mucin depletion (Figure 2E), and severe-mucin depletion (Figure 2F). We demonstrated that the total

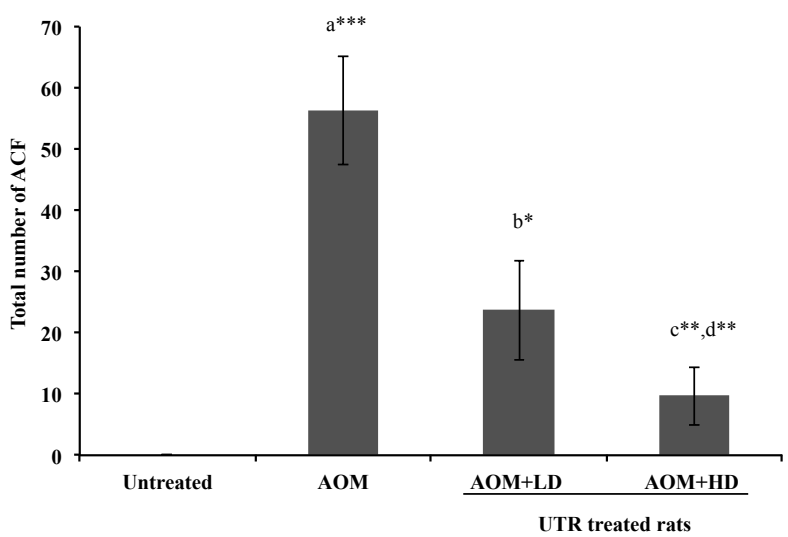

Figure 1. Effect of UTR on total number of ACF in AOM-induced rats. Data were expressed as mean \pm SD. AOM group is the AOM-induced rats without UTR treatment, $\mathrm{AOM}+\mathrm{LD}$ and $\mathrm{AOM}+\mathrm{HD}$ groups represent the AOM-induced rats with $20 \%$ and $70 \%$ of UTR treatment, respectively. apvalue of AOM and untreated groups, bp- and cp-values of $\mathrm{AOM}+\mathrm{LD}$ and $\mathrm{AOM}+\mathrm{HD}$ groups when compared with $\mathrm{AOM}$ group, respectively, dp-value of AOM+HD and AOM+LD groups. $*, * * * * *=$ statistical significance at $\mathrm{p}<0.05, \mathrm{p}<0.01$, and $\mathrm{p}<0.001$, respectively

Table 1. Effects of Dietary UTR on ACF grading and mucin depletion in AOM-induced rats.

\begin{tabular}{lcccccccc}
\hline Groups & \multicolumn{3}{c}{ ACF grading (\%) } & Chi-square & \multicolumn{3}{c}{ Mucin depletion (\%) } & \multirow{2}{*}{ Chi-square } \\
\cline { 2 - 4 } & Hyperplasia & Low-dysplasia & High-dysplasia & & & Non & Mild & Severe \\
\hline AOM & 18.18 & 30.3 & 51.51 & & 15.38 & 46.15 & 38.46 \\
AOM+LD & 21.88 & 43.75 & 34.38 & $\mathrm{a}^{*}$ & 33.33 & 42.86 & 23.81 & $\mathrm{a}^{* *}$ \\
AOM+HD & 42.86 & 35.71 & 21.43 & $\mathrm{~b}^{* * *}, \mathrm{c}^{* *}$ & 42.86 & 42.86 & 14.29 & $\mathrm{~b}^{* * *}$ \\
\hline
\end{tabular}

*AOM group is the AOM-induced rats without UTR treatment, AOM+LD and AOM+HD groups represent AOM-induced rats with $20 \%$ and $70 \%$ of UTR treatment, respectively. ap-value and bp-value of AOM+LD and AOM+HD groups when compared with AOM group, respectively, and $\mathrm{cp}$-value of $\mathrm{AOM}+\mathrm{LD}$ and $\mathrm{AOM}+\mathrm{HD}$ groups. $*, * *, * * *=$ statistical significance at $\mathrm{p}<0.05, \mathrm{p}<0.01$, and $\mathrm{p}<0.001$, respectively 
Achiraya Tammasakchai et al

percentage of mucin depletion in mild- and severe- grades of AOM group was high number at $84.61 \%$. As shown in Table 1, the UTR treated rats at 20\% (AOM+LD group) and $70 \%$ (AOM+HD group) showed significantly decreased the total percentage of mucin depletion at $66.67 \%(\mathrm{p}<0.01)$ and $57.15 \%(\mathrm{p}<0.001)$, respectively. The severe-mucin depleted ACF were also reduced with $23.81 \%$ and $14.29 \%$ of mucin depletion in UTR treated rats with $\mathrm{AOM}+\mathrm{LD}$ and $\mathrm{AOM}+\mathrm{HD}$ groups, respectively. The data can be concluded that UTR treatment may prevent the loss of mucin production in AOM-induced rats.

\section{Cytotoxic effect of UTR extract on CaCo-2 cells}

Cell viability of $\mathrm{CaCo}-2$ cells treated with UTR extract was first checked to confirm cytotoxicity. The IC50 of UTR extract was $5.87 \pm 0.254 \mathrm{mg} / \mathrm{ml}$ with MTT assay at $24 \mathrm{~h}$ (Figure 3 ). The doses of UTR extracts at 5 and $7.5 \mathrm{mg} / \mathrm{ml}$ were designed to determine the effects of

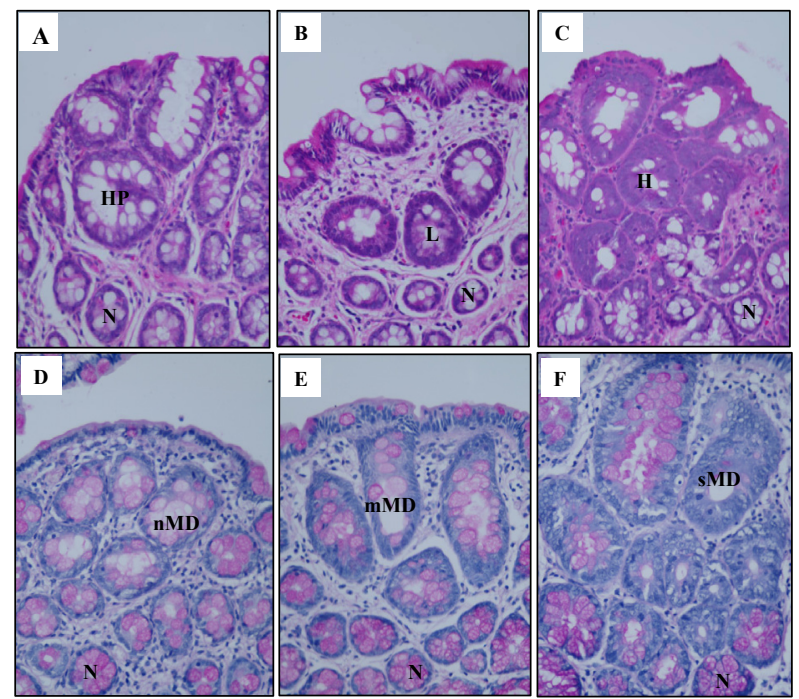

Figure 2. Effects of UTR on dysplastic ACF and mucin production in AOM-induced rats (magnification 400x). $\mathrm{H} \& \mathrm{E}$ staining of ACF with hyperplasia (A), low-grade dysplasia (B), and high-grade dysplasia (C). PAS staining of ACF with non-mucin depletion (D), mild-mucin depletion (E), and severemucin depletion $(\mathrm{F}) . \mathrm{N}=$ normal crypt, $\mathrm{HP}=$ hyperplasia, $\mathrm{L}=$ low-grade dysplasia, $\mathrm{H}=$ high-grade dysplasia. $\mathrm{nMD}, \mathrm{mMD}$, and sMD represent non-mucin depletion, mild-mucin depletion, and severe-mucin depletion in ACF, respectively

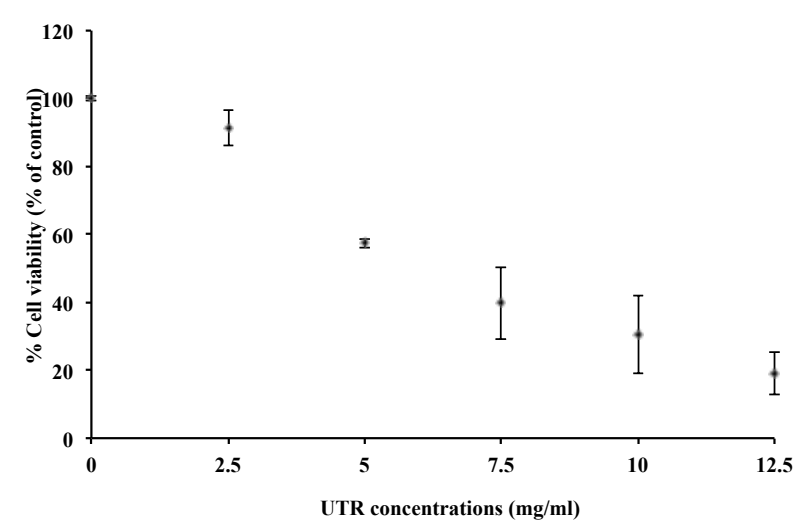

Figure 3. Cytotoxicity of UTR extracts $(2.5-12.5 \mathrm{mg} /$ $\mathrm{ml}$ ) on CaCo-2 cells for $\mathbf{2 4} \mathbf{~ h}$. Untreated group was used as $100 \%$ of control. Data were expressed as mean \pm SD
UTR on apoptotic induction, cellular oxidants, and redox alteration.

\section{UTR extract induced apoptosis in CaCo-2 cells}

The apoptotic cells described with fluorescence staining of the dark blue stained, peripherally clumped, condensed, and fragmented nuclei (Figure 4). Untreated group showed apoptotic population at $10.45 \pm 5.98 \%$ (Figure 4A). Interestingly, the percentage of apoptotic cells in UTR treatment at $5 \mathrm{mg} / \mathrm{ml}$ and $7.5 \mathrm{mg} / \mathrm{ml}$ groups were significantly increased with dose dependent manner at $49.94 \pm 12.63 \%, \mathrm{p}<0.01$ (Figure 4B) and $59.74 \pm 0.83 \%$, $\mathrm{p}<0.01$ (Figure 4C), respectively. On the basis of these results, it can be concluded that the UTR extract can induce the apoptosis in colon cancer cells.

UTR treatment induced cellular oxidants in $\mathrm{CaCo}-2$ cells

The effect of UTR extract on cellular oxidants is shown in Figure 5. UTR treated cells at 5 and $7.5 \mathrm{mg} /$ $\mathrm{ml}$ increased cellular oxidants with DCF fluorescence intensity at $111.43 \pm 0.85 \%(\mathrm{p}<0.001)$ and $120.96 \pm 1.54 \%$ $(\mathrm{p}<0.001)$, respectively when compared with untreated cells. These results can be indicated that the increased cellular oxidants in UTR treated cells may associate with the tendency of UTR induced apoptosis.

UTR treatment altered the GSH/GSSG ratio in CaCo-2 cells

In this study, we investigated the concentrations

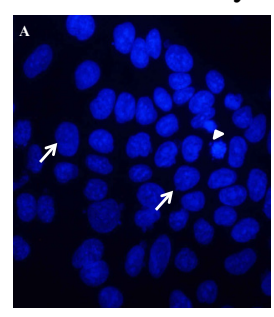

Untreated

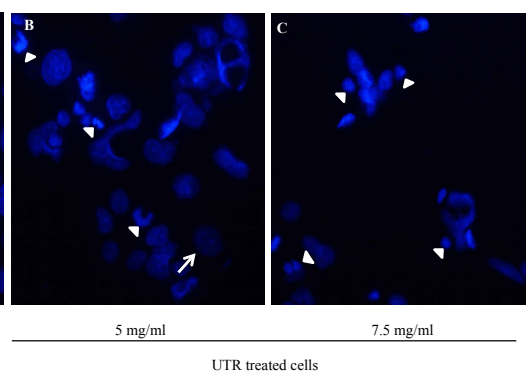

Figure 4. Effects of UTR Extract on Apoptotic Induction in CaCo-2 Cells. Fluorescence staining with Hoechst 33342: apoptotic cells (arrow head), normal nuclei (arrow). All data presented with magnification 200x under fluorescence microscopy

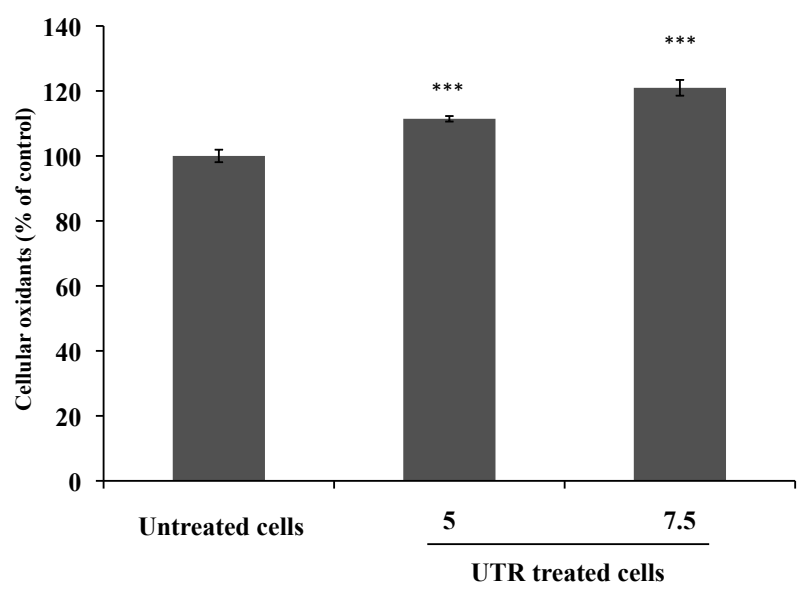

Figure 5. Effect of UTR on Cellular Oxidants in CaCo2 Cells. Data were expressed as mean \pm SD. ***statistical significance at $\mathrm{p}<0.001$ when compared with untreated group 
Unpolished Thai Rice Prevents ACF Formation and Dysplastic Progression in AOM-Induced Rats and Induces Apoptosis

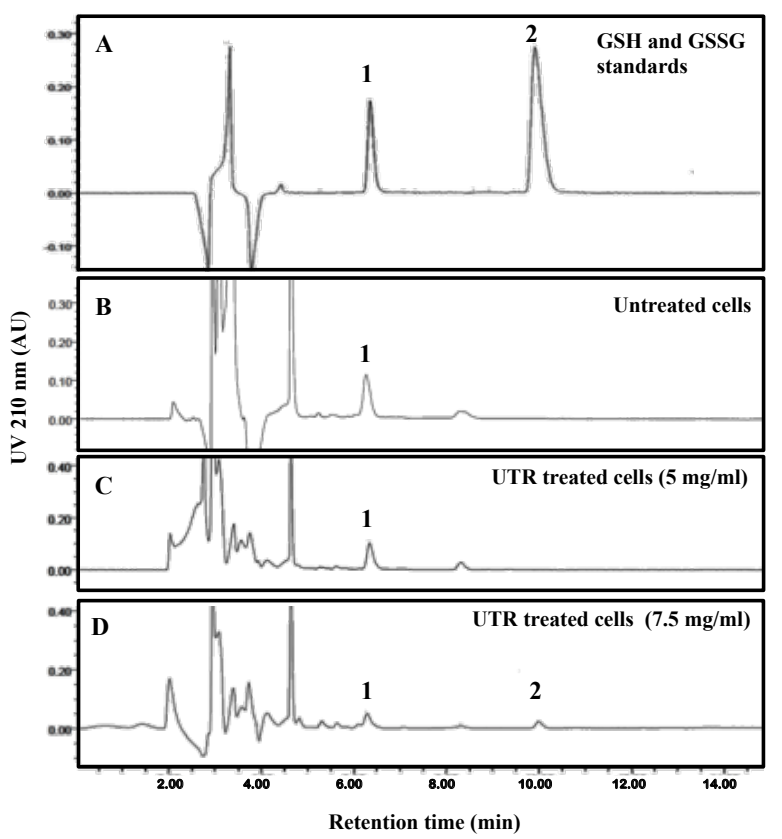

Figure 6. HPLC Chromatogram of GSH and GSSG in UTR Treated CaCo-2 Cells. The references of GSH (1) and GSSG (2)

Table 2. Effect of UTR Extract on GSH and GSSG Levels in CaCo-2 Cells

\begin{tabular}{lll}
\hline Test & \multicolumn{2}{c}{ Glutathione level (Mean \pm SD) } \\
\cline { 2 - 3 } & GSH $(\mathrm{mM})$ & GSSG $(\mathrm{mM})$ \\
\hline Untreated cells & $0.958 \pm 0.022$ & - \\
Treated cells $(5 \mathrm{mg} / \mathrm{ml})$ & $0.519 \pm 0.193 \mathrm{a}^{*}$ & - \\
Treated cells $(7.5 \mathrm{mg} / \mathrm{ml})$ & $0.292 \pm 0.037 \mathrm{~b}^{* *}$ & $0.070 \pm 0.010$
\end{tabular}

*p-value and bp-values of 5 and $7.5 \mathrm{mg} / \mathrm{ml}$ of treated groups, respectively when compared with untreated cells, $*$ and $* *=$ statistical significance at $\mathrm{p}<0.05$ and $\mathrm{p}<0.01$, respectively, $-=$ Undetectable level

of intracellular glutathione in reduced (GSH) and oxidized (GSSG) forms by using HPLC technique. The chromatograms of GSH and GSSG are shown in Figure 6A, the GSH and GSSG were obtained at 6.3 and 9.9 min, respectively. UTR treated cells had a statistically significant reduction with GSH level in a dose dependent manner (Figures 6B, 6C, and 6D), the UTR treatment at 5 and $7.5 \mathrm{mg} / \mathrm{ml}$ showed GSH levels at $0.519 \pm 0.193$ and $0.292 \pm 0.037 \mathrm{mM}$, respectively. Interestingly, GSSG was found only in UTR treatment at a dose of $7.5 \mathrm{mg} / \mathrm{ml}$ $(0.070 \pm 0.010 \mathrm{mM})$. However, the increasing of GSSG showed inversely correlated with the GSH level (Table 2). The results indicated that UTR treatment may induce cellular redox alteration by a decreased GSH/GSSG ratio. This alteration may induce a cellular oxidative stress relating apoptosis in colon cancer cells.

\section{Discussion}

Aberrant crypt foci (ACF) formation is well recognized to associate with precancerous lesion in colorectal cancer (CRC) development (Archer et al., 1992; Thorup, 1997). In this study, we demonstrated that dietary red color strain of unpolished Thai rice (UTR) can reduce total number of ACF in AOM-induced rats with a dose dependent manner. This finding is in agreement with previous reports, purple rice extract had a positive to prevent ACF formation in Dimethyhydrazine (DMH)-induced rats (Summart and Chewonarin, 2014). Phenolic-rich plants are strongly associated with the prevention of colorectal cancer (CRC) (Pandey KB and Rizvi SI, 2009). Polyphenols were significantly and dose-dependently decreased total number of ACF in rats (Xiao et al., 2008). The grapederived polyphenols showed a reduced crypt multiplicity (Campanholo et al., 2015) and ACF formation in rats (Walter et al., 2010). In addition, they also inhibited tumor growth and tumor vascularization (Walter et al., 2010).

Generally, the high grade dysplastic ACF have been suggested to be more relevant precancerous lesions to develop into cancer, whereas hyperplastic ACF are not (Barthold, 1981; Cardiff et al., 2006). In this study, the AOM-induced rats without UTR treatment showed high percentage of the high-grade dysplasia. However, the dietary UTR treatment groups showed significantly decreased the number of high-grade dysplastic ACF in a dose dependent manner. Thus, dietary UTR may be beneficial for the inhibition in precancerous stage relating cancer development. The severe grade of mucin depletion has been closely related to CRC development (Caderni et al., 2003). In this study, dietary UTR showed significantly reduced the percentage of mucin depletion in a dose dependent manner. The results may be concluded that the UTR treatment can prevent mucin depletion in ACF by maintaining the normal function of mucin production in colonic epithelium. This finding was supported by previous study, the rice bran oil had strongly reduced the number of mucin-depleted ACF (Shih et al., 2011).

This study, UTR extract showed dose-dependently increased the percentage of apoptotic cells in CaCo-2 cells at treated dose 5 and $7.5 \mathrm{mg} / \mathrm{ml}$. The high number of apoptotic cancer cells had associated with the increased of cellular oxidants. Previously, an excess of oxidative stress in cancer cells can induce cell cycle arrest and/or apoptosis (Wondrak et al., 2009). In this study, we found that UTR extract had significantly induced cellular oxidants in a dose dependent manner. This finding is in agreement with previous studies, various phytophenolics such as EGCG, curcumin, quercetin, and gallic acid can be generated ROS with dose- and time-dependent manners in various cancer cells (Kelly et al., 2001; Long et al., 2002).

An intracellular redox balance, especially GSH/GSSH ratio is an important role in cellular oxidative stress response (Noda et al., 2001). Cellular stress has been associated with the potential function for cell proliferation and apoptosis (Aw, 2003). In this study, UTR showed significantly decreased GSH level in a dose dependent manner. The result related to previous studies, resveratrol activated GSH efflux and it can induce apoptosis in cells (Guha et al., 2011). Grape seed extract showed not only induced oxidative stress via superoxide formation but it also reduced intracellular GSH level in cancer cells (Tyagi et al., 2013). So, UTR may induce cellular oxidants relating apoptotic cells through the decreased ratio of GSH/GSSG.

In conclusion, the UTR showed a beneficial role to prevent $\mathrm{ACF}$ formation, inhibit precancerous progression in AOM-induced rats, and also induce cancer cell 
apoptosis through the alteration of GSH/GSSG ratio. So, UTR may be a candidate for developing pharmaceutical products for CRC prevention.

\section{Acknowledgements}

This study was supported by Mahidol University, Faculty of Science, Mahidol University, and the Thailand Research Fund (A grant number IRG 5780011). We are grateful to Prof. Dr. Nateetip Krishnamra for providing CaCo-2 cells line and to Prof. Dr. Rachanee Udomsangpetch for cell culture facilities. Thanks also go to Department of Pathobiology, Faculty of Science, Mahidol University for providing laboratory space and facilities for this work.

\section{References}

Allen S, Sotos J, Sylte MJ, et al (2001). Use of Hoechst 33342 staining to detect apoptotic changes in bovine mononuclear phagocytes infected with Mycobacterium avium subsp. paratuberculosis. Clin Diagn Lab Immunol, 8, 460-4.

Archer MC, Bruce WR, Chan CC, et al (1992). Aberrant crypt foci and microadenoma as markers for colon cancer. Environ Health Perspect, 98, 195-7.

Aw TY (2003). Cellular redox: a modulator of intestinal epithelial cell proliferation. News Physiol Sci, 18, 201-4.

Basu S, Roychoudhury A, Danyal S, et al (2012). Carbohydrate content and antioxidative potential of the seed of three edible indica rice (Oryza sativa L.) cultivars. Indian J Biochem Biophys, 49, 115-23.

Barthold SW (1981). Modification of early dimethylhydrazine carcinogenesis by colonic mucosal hyperplasia. Cancer Res, 40, 4451-5.

Bird RP (1995). Role of aberrant crypt foci in understanding the pathogenesis of colon cancer. Cancer Lett, 93, 55-71.

Bird RP, Good CK (2000). The significance of aberrant crypt foci in understanding the pathogenesis of colon cancer. Toxicol Lett, 15, 395-402.

Caderni G, Femia AP, Giannini A, et al (2003). Identification of mucin-depleted foci in the unsectioned colon of azoxymethanetreated rats: correlation with carcinogenesis. Cancer Res, $\mathbf{6 3}$, 2388-92.

Campanholo VM, Silva RM, Silva TD, et al (2015). Oral concentrated grape juice suppresses expression of NF-kappa B, TNF- $\alpha$ and iNOS in experimentally induced colorectal carcinogenesis in Wistar Rats. Asian Pac J Cancer Prev, 16, 947-52.

Cardiff RD, Anver MR, Boivin GP, et al (2006). Precancer in mice: animal models used to understand, prevent, and treat human precancers. Toxicol Pathol, 34, 699-707.

Circu ML, Aw TY (2012). Glutathione and modulation of cell apoptosis. Biochim Biophys Acta, 1823, 1767-77.

Dickinson DA, Forman HJ (2002). Cellular glutathione and thiols metabolism. Biochem Pharmacol, 64, 1019-26.

Guha P, Dey A, Sen R, et al (2011). Intracellular GSH depletion triggered mitochondrial Bax translocation to accomplish resveratrol-induced apoptosis in the U937 cell line.J Pharmacol Exp Ther, 336, 206-14.

Hahn H, Huck CW, Rainer M, et al (2007). Analysis of glutathione in supernatants and lysates of a human proximal tubular cell line from perfusion culture upon intoxication with cadmium chloride by HPLC and LC-ESI-MS. Anal Bioanal Chem, 388, 1763-9.

Halliwell B (1994). Free radicals, antioxidants, and human disease: curiosity, cause or consequence?. Lancet, 344, 721-24.

Kelly MR, Xu J, Alexander KE, et al (2001). Disparate effects of similar phenolic phytochemicals as inhibitors of oxidative damage to cellular DNA. Mutat Res, 485, 309-18.

Khan M, Li T, Khan MKA, et al (2013). Alantolactone induces apoptosis in HepG2 cells through GSH depletion, inhibition of STAT3 activation, and mitochondrial dysfunction. Biomed Res Int, 2013, 719858 .

Lobo V, Patil A, Phatak A, et al (2010). Free radicals, antioxidants and functional foods: Impact on human health. Pharmacogn Rev, 4, 118-26.

Long LH, Clement MV, Halliwell B (2000). Artifacts in cell culture: rapid generation of hydrogen peroxide on addition of (-)-epigallocatechin(-)-epigallocatechin gallate(+)-catechin, and quercetin to commonly used cell culture media. Biochem Biophys Res Commun, 273, 50-3.

Loo G (2003). Redox-sensitive mechanisms of phytochemicalmediated inhibition of cancer cell proliferation (review). J Nutr Biochem, 14, 64-73.

Noda T, Iwakiri R, Fujimoto K, et al (2001). Induction of mild intracellular redox imbalance inhibits proliferation of $\mathrm{CaCo}-2$ cells. FASEB J, 15, 2131-9.

Pandey KB, Rizvi SI (2009). Plant polyphenols as dietary antioxidants in human health and disease. Oxid Med Cell Longev, 2, 270-8.

Pelicano H, Carney D, Huang P (2004). ROS stress in cancer cells and therapeutic implications. Drug Resist Updat, 7, 97-110.

Perše M (2013). Oxidative stress in the pathogenesis of colorectal cancer: cause or consequence?. Biomed Res Int, 2013, 1-9.

Rattanachitthawat S, Suwannalert P, Chaiyasut C, et al (2010). Phenolic content and antioxidant activities in red color of unpolished thai rice prevent oxidative stress in rats. $J$ Med Plants Res, 4, 796-801.

Shahidi F, Wanasundara PK (1992). Phenolic antioxidants. Crit Rev Food Sci Nutr, 32, 67-103.

Shih CK, Ho CJ, Li SC, et al (2011). Preventive effects of rice bran oil on 1,2-dimethylhydrazine/dextran sodium sulphate-induced colon carcinogenesis in rats. Food Chem, 126, 562-7.

Summart R, Chewonarin T (2014). Purple rice extract supplemented diet reduces DMH- induced aberrant crypt foci in the rat colon by inhibition of bacterial $\beta$-glucuronidase. Asian Pac J Cancer Prev, 15, 749-55.

Suwannalert P, Rattanachitthawat S, Chaiyasut C, et al (2010). High levels of 25-hydroxyvitamin D3 [25(OH)D3] and $\alpha$-tocopherol prevent oxidative stress in rats that consume Thai brown rice. $J$ Med Plant Res, 4, 120-4.

Tammasakchai T, Reungpatthanaphong S, Chaiyasut C, et al (2012). Red strain Oryza sativa-unpolished Thai rice prevents oxidative stress and colorectal aberrant crypt foci formation in rats. Asian Pac J Cancer Prev, 13, 1929-33.

Thorup I (1997). Histomorphological and immunohistochemical characterization of colonic aberrant crypt foci in rats: relationship to growth factor expression. Carcinogenesis, 18, 465-72.

Traverso N, Ricciarelli R, Nitti M, et al (2013). Role of glutathione in cancer progression and chemoresistance. Oxid Med Cell Longev, 2013, 1-10.

Tyagi A, Raina K, Gangar S, et al (2013). Differential effect of grape seed extract against human non-small-cell lung cancer cells: the role of reactive oxygen species and apoptosis induction. Nutr Cancer, 65, 44-53.

Uchida K, Kado S, Onoue M, et al, (1997). Relationship between the nature of mucus and crypt multiplicity in aberrant crypt foci in the rat colon. Jpn J Cancer Res, 88, 807-14.

Walter A, Etienne-Selloum N, Brasse D, et al (2010). Intake of grapederived polyphenols reduces $\mathrm{C} 26$ tumor growth by inhibiting angiogenesis and inducing apoptosis. FASEB J, 24, 3360-9.

Wondrak GT (2009). Redox-directed cancer therapeutics: molecular mechanisms and opportunities. Antioxid Redox Signal, 11, 3013-69.

Xiao H, Hao X, Simi B, et al (2008). Green tea polyphenols inhibit colorectal aberrant crypt foci (ACF) formation and prevent oncogenic changes in dysplastic ACF in azoxymethane-treated F344 rats. Carcinogenesis, 29, 113-9. 